\title{
ENERGY EFFICIENT WIRELESS SENSOR NETWORKS
}

\author{
Amin M. Abbosh ${ }^{(1)}$ and David V. Thiel ${ }^{(2)}$, Senior Member, IEEE \\ ${ }^{(1)}$ School of ITEE, The University of Queensland, QLD 4072, Australia \\ ${ }^{(2)}$ Centre for Wireless Monitoring and Applications, Griffith University, QLD 4111, Australia
}

\begin{abstract}
A theoretical model is presented to estimate the energy requirements of multiple-input multiple-output (MIMO) systems when used in wireless sensor networks (WSN) assuming clustered channel modeling, with Rician distribution and cochannel interference. The effect of different propagation environments is simulated. Simulation results show that the single-input singleoutput (SISO) can only be useful with short range systems (less than $20 \mathrm{~m}$ ). The MIMO system with channel state information has the best performance for applications that require a range more than $20 \mathrm{~m}$ and under different propagation conditions. The results presented in this paper indicate that increasing the number of antennas for $M I M O$ systems has a negative effect on the performance under free space propagation, whereas it has a positive effect, especially for MIMO with channel state information (CSI), under "severe" conditions.
\end{abstract}

Index Terms - Energy efficiency, multiple-input multiple-output (MIMO), wireless sensors networks.

\section{INTRODUCTION}

New technologies allow wireless monitoring systems to shrink in size, cost and energy requirements. One objective is to build a complete wireless unit (sensor, RF transceiver, analogue to digital converters ADC, digital to analogue converters DAC and base band processors) in a single chip with limited size. Disposable units can be used in battery powered wireless sensing and monitoring systems. One of the main issues in using those units is how to maximize their operational lifetime.

Recently, the multiple-input multiple-output (MIMO) technique has been proposed to minimize the power requirements of the wireless sensor networks [1]. Results obtained by simulation in [1] show that due to the relatively high power requirements of the additional circuitry, the overall performance of MIMO system was inferior to that of SISO when working in short range applications. All simulations in [1] were carried out assuming a path loss exponent equal to two, i.e. only free space propagation was considered. This point cannot be justified because all measurements, whether indoor or outdoor, show that the square law relation does not give a correct estimation of the path loss. The path loss exponent was shown to vary in the range 2-4 [2]. Also, in [1] the assumed Rayleigh fading distribution does not fit the square law propagation used in the analysis. One important category of MIMO systems, MIMO with channel state information $\left(\boldsymbol{M I M O}_{c s i}\right)$, has not been simulated. The use of such systems may give different results as it has been shown to give higher data rates compared to other MIMO systems, i.e., it needs less power for the same data throughput. Effect of cochannel interference was also neglected.

In this paper, clustered channel modeling is used with a Rician fading to compare the performance of

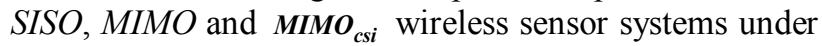
different propagation conditions. The comparison between the different systems assumed a limited array size which is the usual case in wireless sensors. The effect of cochannel interference is also included in the proposed model considering that many wireless systems may be located in a nearby area and some might use the same frequency band.

\section{PROPAGATION MODEL}

The propagation channel model used in this paper is based on the IEEE802.11n clustered channel model. This model describes propagation paths as channel taps grouped in clusters. Each cluster is characterized by a mean angle-of-arrival $(A O A)$ and consists of multiple paths, with $A O A$ values randomly distributed. The power azimuth spectrum $(P A S)$ within each cluster is assumed to have a Laplacian distribution as it coincides with many measurements [3]. The number of clusters, the values of $P A S$ and its standard deviation used in this calculation, are assumed according to model F of IEEE.802.11n [2] as it describes accurately most of the wide area indoor and outdoor environments in which the wireless monitoring system can be used. During simulation, Rician fading is assumed in order to take effect of line-of-sight path into consideration. This is because when WSN work in short range environments there is a high possibility of line of sight propagation.

The first step when simulating the performance of a $M I M O$ system is to find the channel matrix. In this paper we deal only with arrays of length less than one wavelength and the channel matrix $H$ can be found using the following method [4];

$\boldsymbol{H}=\boldsymbol{R}_{r}^{0.5} \boldsymbol{H}_{G} \boldsymbol{R}_{t}^{0.5}$

where $\boldsymbol{H}_{\boldsymbol{G}}$ is the channel matrix with elements that are independent zero mean circularly symmetric complex Gaussian random variables, $\boldsymbol{R}_{\boldsymbol{r}}$ is the $N \times N$ receive covariance matrix and $\boldsymbol{R}_{\boldsymbol{t}}$ is the $M \times M$ transmit covariance matrix, assuming $N$ receive antennas and $M$ transmit antennas. These matrices are calculated using the 
method discussed in [5] after making the following modifications so that it would be suitable for the UCA shown in Fig. 1. Note that the angle of arrival $\phi_{\boldsymbol{U C A}}$ is measured relative to the reference direction defined by elements $i$ and $j$ in the $U C A$;

$\phi_{U C A}=\phi_{U L A}-\left[\frac{2 \pi}{n}(j-i)\right]$

where $n$ is number of elements in the array, $i$ and $j$ are the sequence of elements in the array $(1,2,3 \ldots n)$. Also in this simulation, we will assume a fixed diameter $D$ for the array. Elements of the array are distributed uniformly on the circumference. The spacing $d$ between the $i$ and $j$ elements in the array can be found from the following relation, see Fig.1;

$d=D^{*} \sin \left[\frac{2 \pi}{n}(j-i)\right]$

From the channel matrix, the instantaneous receiver signal to noise ratio $\left(\boldsymbol{S N R _ { i }}\right)$ is calculated using the following equation $[4,5]$;

$S N R_{i}=\frac{\sum_{i=1}^{k} \text { Eigen }_{i}}{M} * \frac{\bar{E}_{b}}{N_{o}}$

where $\bar{E}_{\boldsymbol{b}}$ is the required energy per bit at the receiver to achieve the required bit rate, $\boldsymbol{N}_{\boldsymbol{o}}$ is the noise energy, and Eigen $_{\boldsymbol{i}}$ is the $i^{\text {th }}$ eigenvalue of the channel matrix and $k$ is $\min (N, M)$. Note that for the $\boldsymbol{S I S O}$ system $M=1$ in (4). For $\boldsymbol{M I M \boldsymbol { O } _ { c s i }}$, the water-filling algorithm can be used to maximize the capacity and in this case [5];

$S N R_{i}=\sum_{i=1}^{k} \frac{\bar{E}_{b i}}{M N_{o}}$ Eigen $_{i}$

where $\overline{\boldsymbol{E}}_{\boldsymbol{b} \boldsymbol{i}}$ is the portion of energy allocated to the data transmitted from the $i^{\text {th }}$ antenna.

The two-slope path-loss model [2] is used to calculate the path $\operatorname{loss} \boldsymbol{L}_{\boldsymbol{p}}$. This model assumes that the received power is inversely proportional with the distance squared up to the break-point distance $r_{b r}$. At larger distances $\left(\boldsymbol{r} \gg \boldsymbol{r}_{\boldsymbol{b r}}\right)$ the power falls by a rate proportional to $r^{3.5}$. The break-point distance depends on many parameters including the transmitter antenna height, the receiver antenna height and the wavelength. In this paper $r_{b r}$ is taken to be $10 \mathrm{~m}$ which is suitable for many WSN.

\section{COCHANNEL INTERFERENCE}

Consider a $M I M O$ channel with $M$ antennas at the transmitter, $N$ antennas at the receiver and $K$ cochannel interfering signals each equipped also with $M$ transmitting antennas. The received signal at the desired receiver can be modeled as [6];

$\boldsymbol{y}=\boldsymbol{H}_{d} \boldsymbol{x}_{d}+\sum_{i=1}^{K} \sqrt{\boldsymbol{P}_{i}} \boldsymbol{H}_{i} \boldsymbol{x}_{\boldsymbol{i}}+\boldsymbol{n}$

where $\boldsymbol{H}_{\boldsymbol{d}}$ is the $\boldsymbol{N} \times \boldsymbol{M}$ normalized channel matrix for the desired user, $\mathbf{x}_{\boldsymbol{d}}$ is the $\boldsymbol{M} \times 1$ transmitted signal vector for the desired user. Similarly, $\boldsymbol{P}_{\boldsymbol{i}}$ is the average access path loss of the $i^{\text {th }}$ interferer signal compared with that of the desired signal, $\boldsymbol{H}_{\boldsymbol{i}}$ is $\boldsymbol{N} \times \boldsymbol{M}$ normalized channel matrix of the $i$-th interferer, $\mathbf{x}_{\boldsymbol{i}}$ is the $\boldsymbol{M} \times 1$ transmitting signal vector of the $\boldsymbol{i}^{\text {th }}$ interferer, and $\boldsymbol{n}$ is the additive Gaussian noise vector with zero mean and covariance value $\left(N_{o}\right)$.

The channel matrices $\boldsymbol{H}_{\boldsymbol{d}}$ and $\boldsymbol{H}_{\boldsymbol{i}}$ are formed using the method discussed above. They include the effect of the Rician fading, with a Rice factor $=10 \mathrm{~dB}$ which means a strong line-of-sight ray is present, and transmitter and receiver correlation as well as the effect of mutual coupling between elements of the transmitting and receiving array.

From (6) the mutual information can be calculated using the following equations [7];

$$
I=\log _{2}\left(\operatorname{det}\left(I_{N}+H_{d} R_{d} H_{d}{ }^{H} B_{I}{ }^{-1}\right)\right)
$$

$B_{I}=H_{I} R_{I} P_{I} H_{I}^{H}+N_{o} I_{N}$

$R_{d}=E\left(x_{d} x_{d}{ }^{H}\right)$

where $I_{N}$ is $N \times N$ identity matrix, $H_{I}$ is $N \times N I$ matrix which is result of composition of the interferers matrices $\boldsymbol{H}_{\boldsymbol{i}}$ where the first $M$ columns of $\boldsymbol{H}_{\boldsymbol{I}}$ comes from matrix of the first interferers and the second $M$ columns are channel matrix of the second interferer and so on. $N I$ is the total number of interfering antennas and it is equal to


$\boldsymbol{P}_{\boldsymbol{i}}$ where the first $M$ elements of the diagonal comes from effect of the first interferer and so on. The $N I \times N I$ diagonal matrix $R_{I}$ is formed by the same way from the elements;

$\boldsymbol{R}_{i}=E\left(x_{i} x_{i}{ }^{H}\right)$

The superscript $(\boldsymbol{H})$ in (10) indicates the Hermitian operation.

In this paper, we have assumed that there are many systems working nearby to the system under consideration. Distribution of the systems and frequency allocation were chosen according to the 7-cell reuse system, although any other configuration can be assumed. Therefore, under full load conditions the desired user will suffer from six cochannel interferers [7], i.e. $K=6$.

The following scenarios were considered in this paper. The first one is $M I M O$ without $C S I$ and the power is assumed to be equally divided between elements of the array. Hence, (9) and (10) can be written as;

$\boldsymbol{R}_{d}=\boldsymbol{R}_{1}=\boldsymbol{R}_{2}=\ldots=\boldsymbol{R}_{N I}=\frac{\bar{E}_{b}}{M}$

where $\overline{\boldsymbol{E}}_{\boldsymbol{b}}$ is the total energy per bit transmitted from each sensor.

The position of the desired user is assumed to be random within its cell. Its position relative to the center of the cell is $\boldsymbol{r}_{\boldsymbol{d}}$ where $0 \leq \boldsymbol{r}_{\boldsymbol{d}} \leq \boldsymbol{R}$ assuming that the cell has a radius equal to $R$. The distance between center of the desired cell and center of any of the interfering cells is $D$ and its value can be calculated as $\boldsymbol{D}=\sqrt{21} \boldsymbol{R}$ [7].

Assuming that the $i$-th cochannel sensor is at a distance $r_{i}$ from the center of its cell, the desired sensor and the $i$-th interferer are assumed to be at angles $\theta_{\boldsymbol{d}}$ and $\theta_{i}$ respectively with respect to $D$, (see Fig.2). The 
distance $\boldsymbol{d}_{\boldsymbol{i}}$ between that interferer and the desired sensor can be calculated geometrically. The parameters $r_{d}$ and $r_{i}$ are assumed to take any value between 0 and $R$ with uniform distribution while the parameters $\theta_{\boldsymbol{d}}$ and $\theta_{\boldsymbol{i}}$ can take any value between 0 and $360^{\circ}$ with uniform distribution also. The two-slope path loss model can be used to calculate the path loss between the client and the desired sensor $\left(\boldsymbol{L}_{\boldsymbol{p} d}\right)$ by using the distance $r$ as $\boldsymbol{r}_{\boldsymbol{d}}$, whereas the cochannel interferer path loss, $\boldsymbol{L}_{\boldsymbol{p} i}$ is calculated by replacing $r$ with $\boldsymbol{d}_{i}$. The average access path loss for the $i$-th interferer can now be found as $\left(\boldsymbol{L}_{p d} / \boldsymbol{L}_{p i}\right)$, therefore;

$$
\boldsymbol{P}_{i}=\left(\boldsymbol{L}_{p d} / \boldsymbol{L}_{p i}\right)
$$

In this equation, the same average path loss between any antenna element in transmitter of the interferer and any antenna element in the desired receiver is assumed. This assumption comes from the fact that the effect of fast fading was included in the channel matrix.

The signal to interference and noise ratio (SINR) can be calculated from (7) by summing all the positive eigen values. Using (7), (8), and (11);

$$
\operatorname{SINR}_{\text {MIMo }}=\sum \operatorname{eig}\left(\mathrm{H}_{d} \mathrm{H}_{d}{ }^{H}\left(\mathrm{H}_{I} \boldsymbol{P}_{I} \mathrm{H}_{I}{ }^{H}+M N_{o} / \bar{E}_{b}\right)^{-1}\right)
$$

The second scenario is $\boldsymbol{M I M O}_{\boldsymbol{c s i}}$. In this case the water-filling algorithm can be used, i.e. non-uniform distribution of power between array elements. It has been shown that if the maximum ratio combining $M R C$ is used in the receiver to maximize the SINR then [7];

$$
\operatorname{SINR}_{c s i}=\max \operatorname{eig}\left(\bar{E}_{b} H_{d} H_{d}{ }^{H} B_{I}^{-1}\right)
$$

\section{ELECTRONIC CIRCUITRY}

In the simulation, the SISO transmitter and receiver electronics used are as shown in Fig.3. For the MIMO system there will be $N$ parallel branches of the receiver circuits and $M$ branches of the transmitter circuits as shown in Fig.3. The power requirements for different parts of the electronic circuitry of SISO or MIMO system are as given in [8].

The following equations are used to estimate the energy requirements [1].

$$
\begin{aligned}
\boldsymbol{E}_{b} & =(1+\alpha)\left[\overline{\boldsymbol{E}}_{b} * \frac{M a r * N_{f}}{G_{t} G_{r}} * L_{p}\right]+\frac{\boldsymbol{P}_{c}}{\boldsymbol{R}_{b}} \\
\boldsymbol{P}_{\boldsymbol{c}} & =\boldsymbol{M P _ { t }}+2 \boldsymbol{P}_{s}+\boldsymbol{N} \boldsymbol{P}_{\boldsymbol{r}} \\
\boldsymbol{B}_{e} & =\operatorname{Mean}[Q(\sqrt{2 * S I N R})]
\end{aligned}
$$

where the parameter to be minimized is $\boldsymbol{E}_{\boldsymbol{b}}$, the total energy consumption per bit, $\alpha$ defines the amplification characteristics of the power amplifier at the transmitter, $M_{a r}$ is the safety margin for the system, $N_{f}$ is noise figure of the receiver, $\boldsymbol{G}_{\boldsymbol{t}} \boldsymbol{G}_{\boldsymbol{r}}$ are the gains of an antenna element at the transmitter and receiver side respectively, $d$ is the transmitter-receiver distance, $\lambda$ is the wavelength , $n$ is the path-loss exponent, $\boldsymbol{R}_{\boldsymbol{b}}$ is the bit rate $\boldsymbol{P}_{\boldsymbol{c}}, \boldsymbol{P}_{\boldsymbol{t}}, \boldsymbol{P}_{\boldsymbol{s}}, \boldsymbol{P}_{\boldsymbol{r}}$ are the power consumption values of the circuit sections for one branch of the transmitter digital- to-analog converter, mixer and filter; the synthesizer; and the receiver low-noise amplifier, mixer, intermediate frequency amplifier, filter and analog-to-digital converter. The $Q$ function in (15c) is the Gaussian error integral and $\boldsymbol{B}_{\boldsymbol{e}}$ is the bit error rate. The word (Mean) in (15c) indicates that the estimation should be averaged over all the channel matrix possibilities.

\section{RESULTS AND DISCUSSIONS}

The performance of the systems under consideration is simulated assuming that the frequency of operation is $2.5 \mathrm{GHz}$, maximum bit error rate is 0.001 , and the bit rate is $10 \mathrm{Kbps}$. Channel simulations assume Rician distribution with a rice factor of $10 \mathrm{~dB}$. Elements of the $M I M O$ are distributed uniformly on the circumference of the circle $\lambda / 2$ diameter value.

The results introduced in Figs. 4 and 5 shows a comparison between the energy needed when using SISO, $M I M O$ and мIмо $_{c s i}$ under different propagation conditions. It is clear that the SISO system needs minimum energy for operation over very short distances, i.e. less than $20 \mathrm{~m}$. This is because at short distances most energy is needed for the electronic circuitry. As the SISO has the minimum hardware, then it is expected that it will require little energy. The next best system is the $\boldsymbol{M I M O}_{c s i}$ when using a two-antenna array. This is mainly because this system chooses propagation through a channel that gives it the highest possible $S N R$. The four-antenna array the $M I M O$ system requires more hardware and so more energy to activate and when the distance is short enough the energy needed by the circuitry will be a major part in the overall energy requirement. As the distance increases, the energy needed to compensate for the path loss increases until it dominates the total energy requirement. At that distance $\boldsymbol{M I M O}_{\text {csi }}$ will be the preferred system. Under a severe propagation condition, i.e. when the path loss exponent increases, the energy needed to overcome the increased path loss will be high compared to the case of a soft propagation condition. In this case the ability of the $M I M O$ system in difficult propagation situations appears more obvious and the use of a four-antenna array improves the performance even more.

\section{CONCLUSION}

A theoretical model has been presented to estimate the energy requirements of $M I M O$ systems when used in wireless sensor networks (WSN) assuming clustered channel modeling, with Rician distribution and cochannel interference. The effect of different propagation environments has been simulated. Results from the simulation have shown that the SISO is only useful with short range systems (less than 20m). The MIMO system with channel state information has the best performance for applications that require range more than $20 \mathrm{~m}$ and under different propagation conditions. The results presented in this paper have indicated that increasing the number of antennas for $M I M O$ systems has a negative effect on the performance under free space propagation, 
whereas it has a positive effect, especially for MIMO with channel state information, under "severe" conditions.

\section{ACKNOWLEDGMENTS}

A. Abbosh acknowledges the financial support of the University of Queensland via ECR grant.

\section{REFERENCES}

[1] S. Cui, A. Goldsmith and A. Bahai, "Energy-efficiency of MIMO and cooperative MIMO Techniques in sensor networks", IEEE Journal on Selected Areas in Comm., vol.22, no.6, pp. 1089-1098, 2004.

[2] V. Erceg et .al, "TGn channel models," IEEE 802.11 document 03/940r4, May 2004.

[3] K. Pederson, P. Mogensen, and B. Fleury, "Spatial channel characteristics in outdoor environments and their impact on BS antenna system performance," Proc. Of IEEE Vehicular Tech. Conf., Canada, vol.2, 1998, pp. 719-723.

[4] J. Kermoal, L. Schumacher, K. Pedersen, and P. Mogensen, "A stochastic MIMO radio channel model with experimental validation," IEEE Journal on Selected areas on Comm., vol.20, no.6, pp. 1211-1226, 2002.

[5] L. Schumacher, K. Pedersen, and P. Mogensen, "From antenna spacings to theoretical capacities- guidelines for simulating spatial correlation in MIMO systems," Proc. Of the $13^{\text {th }}$ IEEE Int. Symp. on Personal Indoor Mobile and Radio Comm., Portugal, 2002.

[6] M. Kang, L. Yang, and M. Alouini, "Certain computations involving complex Gaussian Matrices with applications to the performance analysis of MIMO systems," Chapter-3 in Space-Time Processing for MIMO Communications, John Wiley \& Sons, Canada, 2005.

[7] B. Walke, Mobile radio networks; Networking, protocols and traffic performance, John Wiley \& Sons, 2nd Edition, New York, 2002.

[8] S. Cui, A. Goldsmith, and A. Bahai, "Energy-constrained modulation optimization," IEEE Trans. Wireless Communication, vol.5, pp.2349-2360, 2005.

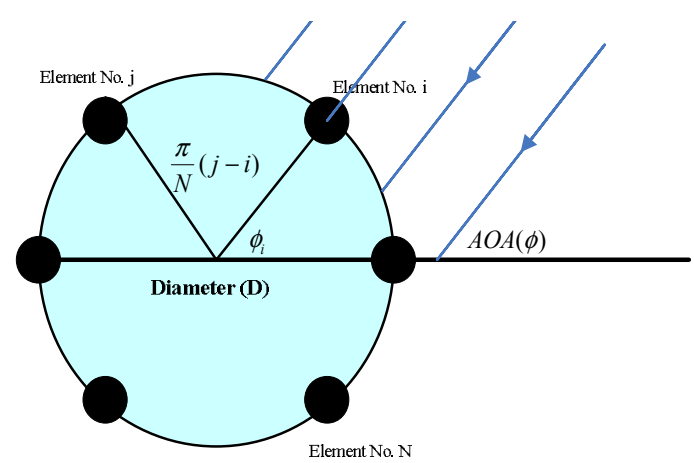

Fig.1 Uniform circular array configuration.

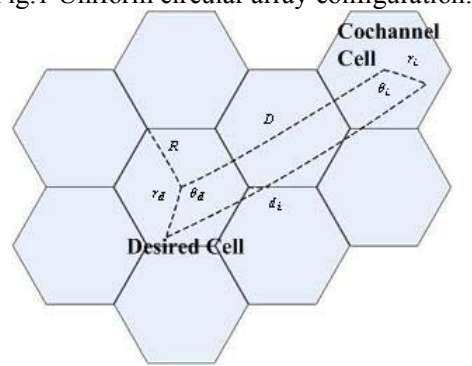

Fig.2 Schematic diagram showing position of the desired sensor and the cochannel interferer, assuming a 7-cell reuse distribution.
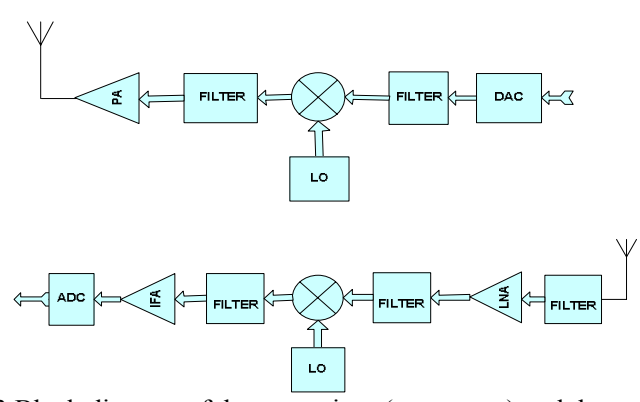

Fig.3 Block diagram of the transmitter (upper part) and the receiver (lower part).

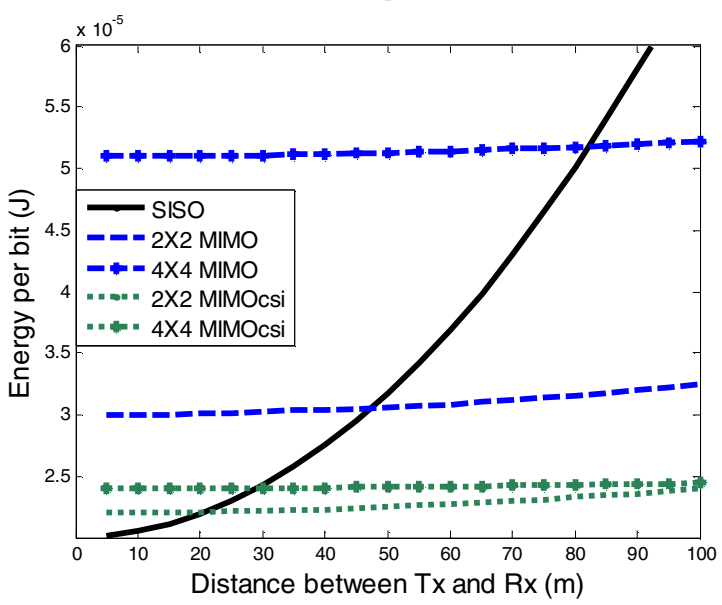

Fig.4 Comparison between the energy required by different systems, assuming free space propagation.

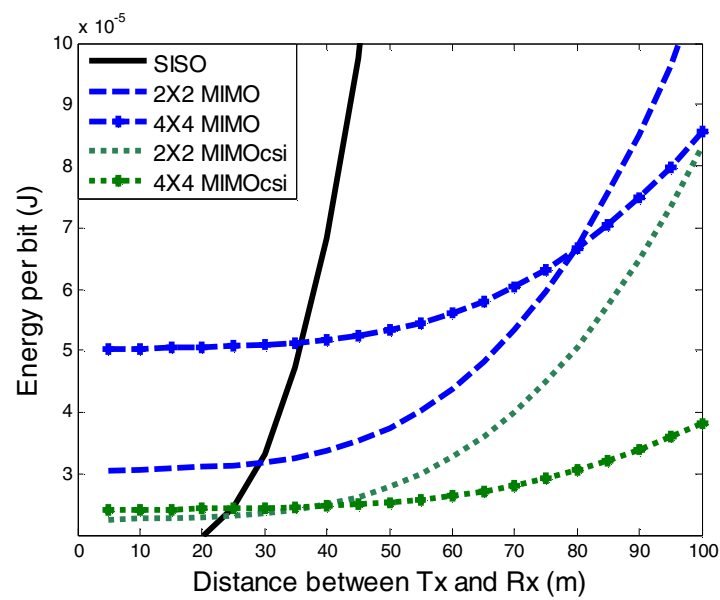

Fig. 5 Comparison between the energy required by different systems, assuming a two-slope propagation model. 